AIAA Space 2011

\title{
Low-Latency Lunar Surface Telerobotics from Earth-Moon Libration Points
}

\author{
Daniel Lester \\ University of Texas, Austin TX 78712 \\ Harley Thronson ${ }^{2}$ \\ NASA Goddard Space Flight Center, Greenbelt MD 20771
}

\begin{abstract}
Concepts for a long-duration habitat at Earth-Moon L1 or L2 have been advanced for a number of purposes. We propose here that such a facility could also have an important role for low-latency telerobotic control of lunar surface equipment, both for lunar science and development. With distances of about $60,000 \mathrm{~km}$ from the lunar surface, such sites offer light-time limited two-way control latencies of order $\mathbf{4 0 0} \mathbf{m s}$, making telerobotic control for those sites close to real time as perceived by a human operator. We point out that even for transcontinental teleoperated surgical procedures, which require operational precision and highly dexterous manipulation, control latencies of this order are considered adequate. Terrestrial telerobots that are used routinely for mining and manufacturing also involve control latencies of order several hundred milliseconds. For this reason, an Earth-Moon LI or $L 2$ control node could build on the technology and experience base of commercially proven terrestrial ventures. A lunar libration-point telerobotic node could demonstrate exploration strategies that would eventually be used on Mars, and many other less hospitable destinations in the solar system. Libration-point telepresence for the Moon contrasts with lunar telerobotic control from the Earth, for which two-way control latencies are at least six times longer. For control latencies that long, telerobotic control efforts are of the "move-and-wait" variety, which is cognitively inferior to near real-time control.
\end{abstract}

\section{Introduction}

$\mathrm{T}$ HE existing paradigm for space robotics is presently double-edged. In one case, we send robotic spacecraft to distant locations where, commanded from the Earth, they execute their tasks, make their observations, and transmit these findings back to Earth where those accomplishments and observations are processed. Armed with these results, a new command set is created and uploaded that allows progress to be made iteratively. Most of space science is currently done in this way. In the other case, robots are seen as local extensions of human dexterity and perception. For the space shuttle and ISS, Canadarms, the Special Purpose Dexterous Manipulator (Dextre, SPDM) and the JEM-RMS are robotic facilities that allow astronauts on-site to manipulate local assets in a way that does not require risky and time-consuming EVA. For the Constellation program, robots just outside a lunar surface habitat were seen as a powerful extension of human reach, again mitigating the need for EVA. Astronauts deep inside the lunar gravity well would operate robots which were functioning in the immediate vicinity.

Placing humans on the lunar surface to operate these robots involves special vehicles, and unique power and life support systems for astronaut habitats. This would be especially costly, whereas the light-time limited control latency or time delay they would have there would be negligible, although available over only distances within direct line of sight. In the case of distant robots, system control can be relatively inexpensive, but involves latencies that can range upwards from several seconds for the Moon. We propose on-orbit telerobotics as a third paradigm for human-robot cooperation that achieves low communication latency without the expense of putting humans in deep gravity wells. It offers new goals for human space flight in the near term. With this strategy, astronauts are not sent

Research Fellow, Department of Astronomy C1400, University of Texas, Austin TX 78712

${ }^{2}$ Senior Scientist for Advanced Concepts, Astrophysics Division, Code 660, Science and

Exploration Directorate, NASA Goddard Space Flight Center, Greenbelt, MD 20771

$$
1
$$


deep into a gravity well, but are stationed just outside of it in free space, close enough to the destination that control latency can be minimized and near telepresence can be achieved.

In the case of high-latency telerobotic control, special measures are taken to try to mitigate latency. This is largely done in software by giving the telerobots a substantial degree of autonomy. Such autonomy is especially challenging for complicated environments like planetary surfaces. In our on-orbit telerobotics approach, latency is mitigated by largely removing it, at least for the humans doing the controlling. In doing so, this on-orbit approach meets a number of current needs. It provides enabling capabilities for many kinds of exploration. It involves no near-term human surface operations, keeping humans out of expensive gravity wells. It builds strongly on investments in and capabilities of terrestrial commercial telerobotics, which are based on negligible latency. It contributes to the demonstration of long-duration human habitation beyond Earth orbit. It is strategically extensible to many destinations, including ones that are very hostile to humans and, perhaps most importantly, can be carried out in the near term. For the lunar surface, this strategy could be one of the first uses of a habitation facility at EarthMoon L1 or L2.

\section{Criticality of Latency for Human Exploration}

Communication latency - round-trip light travel time plus delays in network processing - is one of the most important handicaps to human functionality in and across deep space. In many respects, it is why we want to send people to exploration sites, rather than robots. Throughout our solar system, telerobots controlled from the Earth, by explorers on the Earth, have to endure significant latencies. For the Moon, the two-way latency is at least 2.6 seconds. For Mars, the latency is at least $8-40$ minutes, depending on orbital location. For Voyager, now approaching the edge of our solar system, the control latency is presently around 32 hours. Low latency allows for a telerobotic control mode that we call "telepresence" or, with minimal autonomy, "telesupervision". This is most effective if the latency is smaller than the human cognitive time scale. When the two-way communication latency to a site exceeds our cognitive timescale, the human brain works differently, dropping into a "move-and-wait" mode in order to avoid instability of the human servo mechanism. In this respect, latency impacts our perception of causality. This is well understood from neuroscience, experimental psychology, robotic engineering, and what has been termed "engineering psychology." Some of the first such work of relevance to space exploration was funded by NASA ${ }^{1,2}$, with early work reviewed by Sheridan ${ }^{3}$. Many contemporary considerations of the effect of time delays on human perception and response have been reported ${ }^{4,5,6}$. From the latter two references in particular, we can envisage a kind of sliding scale of autonomy in telesupervision. This is where low latency allows efficient coordination of humans and robots for task sharing, and in which difficult tasks requiring a high level of human judgment and cognition can be handled by telepresence, whereas simpler tasks easily performed autonomously can be done so.

Essentially nothing that we presently do in deep space right now can be considered to approximate real telepresence, though adaptation to large latencies can help robot operators approach that kind of sensibility. Although the Mars Exploration Rovers (MERs) have been run very much in a fragmented communication mode, where iterations between uploaded commanded task sets were done on timescales of many hours, the controllers of Spirit and Opportunity found themselves deeply immersed in the discovery process, and could imagine that they were really on the Martian surface. This psychology was described by several observers ${ }^{7,8}$. The MER scientists were convinced that their work embraced the spirit of in-situ exploration, using machines as surrogates. Whereas the sense of intimate involvement was thrilling, the communication latency was much longer than our cognitive timescale, and made this sense of telepresence a very low-quality one.

What then is the human cognitive timescale? Many detailed studies of the impact of latency on human capabilities have been undertaken, but those impacts are highly task dependent. Some notional numbers are helpful. High-performance online gaming depends on latencies being less than $100-200 \mathrm{~ms}$. Such gaming becomes much less entertaining if the latency exceeds these levels. But other terrestrial pursuits are also relevant to the question.

Telerobotic surgery is a rapidly developing enterprise, where surgeons can use robotic dexterity to increase the precision and stability of their hand motions. Such surgery, it has been realized, can be carried out over large distances, and surgical trials involving cutting, stitching, and tying knots have been completed on transcontinental scales. These trials have shown conclusively that two-way communication latencies of a few hundred milliseconds does not seriously impact surgical success, though latencies over 500 milliseconds make for considerable difficulty. 10,1 Lessons from terrestrial telerobotic surgery should figure strongly in considerations for space 
telerobotics, in that surgery exemplifies very detailed work, requiring a high degree of dexterity and situation awareness, in a very complex, high-value physical environment. An illustrative test of dexterous capability in surgery is laproscopic knot tying. Whereas such a task can be completed telerobotically in well under a minute for latencies of less than 400 milliseconds ${ }^{i 2}$ it appears to require as much as ten minutes for latencies of only about 2 seconds. Although telerobotic surgery is of great interest with regard to human space flight for health maintenance of astronauts, it also should also strongly inform other space telerobotic efforts, and we describe those other efforts here in some detail.

Terrestrial robotic mining operations such as driving bulldozers, and operating drills and grinders, are commonly used in industry, and in many respects duplicate the kind of bulk development operations that would be needed on lunar or planetary regolith. Such terrestrial telerobotic mining operations are routinely used at distances of thousands of kilometers, with two-way latencies (dominated by networking delays) of order 500 milliseconds. ${ }^{13}$ Undersea telerobotics are common for science, oil and gas resource development, and telecommunication cable management. Such latencies are representative for those applications as well, though they are achieved mainly through hardwired communication links. Military drone piloting is done with latencies that can exceed a full second, but just flying is not a time-critical activity, and targeting and landing are tasks that are given some degree of autonomy. There are many commercial applications for telerobotic surveillance using similarly small time delays, such as for search and rescue operations. Those do not demand the lowest operational latency, though often have to be guided through environments with challenging topology. ${ }^{14}$ Finally, it should be noted that the human reaction time for visual stimulus is about 200 milliseconds, and a bit shorter for audio stimulus. The human brain cannot process information and react to it much faster than this. We can confidently assume here that the cognitive timescale for a human being is on this order, of 500 milliseconds. This is considerably shorter than the two-way time delay from the Earth to Moon, though is of the same order as for, say, Earth to geosynchronous satellites, which may be a fruitful venue for telepresence servicing. We thus conclude that for telerobotic operations on the Moon and farther, high-quality cognitive telepresence or telesupervision is not possible from the Earth, at least to the extent that challenging dexterous manipulation or control is involved.

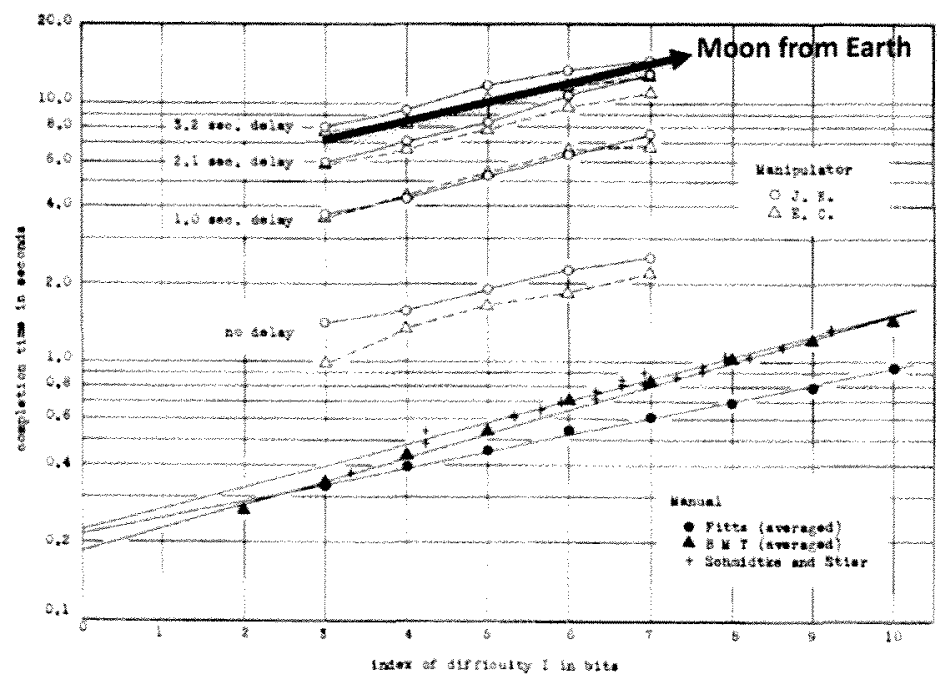

Figure 1. Task completion time as a function of latency. Adapted from Ferrell ${ }^{2}$. This plot shows the time required to telerobotically grasp a target and move it in a two-dimensional frame as a function of latency. The "difficulty index" is proportional to the distance over which the telerobotic "fingers" have to be moved. The "manual" points at the bottom of the plot are where the task is completed without telerobotics. The task completion time corresponding to a 2.6 second two-way latency for the Moon, as controlled from Earth, has been added to the plot near the top. Even for simple tasks like this, the latency cost can be considerable.

A cognitive timescale of 500 milliseconds corresponding to a two-way light-time latency leads naturally to what we can call a cognitive distance scale, or cognitive horizon, of 250 milliseconds $\mathrm{x} c=75,000 \mathrm{~km}$. Beyond this distance, the speed of light makes telerobotic control a highly non-cognitive enterprise, and robotic autonomy becomes an important factor in ensuring performance. At less than this distance, a human being can plausibly achieve high-quality telepresence. Although autonomy can still be very helpful, a human being within this distance can, in principle, perform cognitively as well as he or she could being physically at the exploration site. LEO is clearly within the cognitive horizon for terrestrial controllers, as is GEO, so these are regimes where high-quality telepresence for Earth-based controllers is obviously achievable. In these cases, cognitive functionality is, in practice, limited mainly by networking delays. For example, whereas TDRSS provides high-bandwidth communication for near-Earth facilities, it offers very poor latency compared to the human cognitive timescale. The Moon, as noted above, is well beyond this cognitive horizon for controllers on the Earth. That does not mean that

3 
controllers on the Earth cannot teleoperate equipment on the Moon, but that doing so requires additional time. That additional time may very substantially exceed the formal time delay. The extent to which it does will depend on the specific task, but early work on this subject is instructive.

Figure 1 shows the results from Ferrell ${ }^{2}$, in a famous early laboratory study of the cognitive effects of latency. The open points are those determined using a "minimal manipulator" setup, where an operator uses potentiometers to control, over a specified delay, the two-dimensional position of a slave "hand." The operator was instructed to move the slave hand to where "fingers" on it, when closed, would grasp a small block. The "difficulty index" was proportional to the distance over which the "hand" had to travel to get to the block. These results are compared, in the solid points, to various human motor tests which had a hand simply touch or pick up a target and move it to a nearby slot. It is clear that even for this very simple, indeed trivial action, the task completion time far exceeds the latency involved. In fact, if this task had been done telerobotically on the Moon, commanded from the Earth, it would have taken approximately ten seconds, about eight times longer than if it had been done telerobotically from a nearby lunar habitat with no delay, and about twenty times longer than if done directly by an astronaut on the Moon (albeit without the confining and restrictive handicap of a space suit). Surgical trials have not been done for latencies longer than about a second, but suggest a rapidly rising task completion time, longer than the time delay This suggests that the time handicap for task completion is not, in the case of a complicated task, simply proportional to the latency, but increases much faster than the latency alone.

A pioneering space telerobotic experiment called ROTEX was carried out by the DLR on the space shuttle in $1993 .^{15}$ ROTEX was a small 6-axis telerobot that was equipped with a number of dexterous implements and sensors. This was controlled both by astronauts on board, with minimal latency, and from the ground via TDRSS with a 6.5 second two-way communication latency. While simple tasks were achieved from ground control, ROTEX was used in at least partly an autonomous way, based on predictive simulation. With this heritage, we look forward to the activation of the ambitious "R2" NASA/GM humanoid telerobot now at ISS. With the same TDRSS latency restrictions as for ROTEX however, R2 will be used from the ground, in this venue, in a supervised autonomy mode, rather than fully immersive telepresence. In principle, however, such a robot could be used on the Moon with a highquality telepresence if the control latency could be reduced to the cognitive timescale of human operators.

We note that such an estimate for a cognitive horizon in space is applicable to mainly what we may refer to as visual cognition, or visual modality. The effects of latency on haptic modality are more serious than for visual modality, and that delays of less than 100 milliseconds can seriously compromise the haptic perception of a surface. ${ }^{16.17}$ To the extent that the human sense of touch on other worlds will already be seriously compromised by heavy space suit gloves, however, latency may not be a relative handicap for remote operators.

\section{On-Orbit Control Venues}

A recognized venue for discovery, exploitation, and human shelter in space is the Moon. The Apollo missions demonstrated some of what humans could accomplish there, but NASA's present plans for human space flight do not include human return, at least in the near term. In large measure, those plans are restricted because of the expense of landing people on the Moon, and support for them there in environments that are in some respects (e.g. dust, gravity) more hostile than in free space. The Augustine Committee ${ }^{18}$ understood that sending humans into deep gravity wells was a particularly costly proposition, and developed a "flexible path" policy that would have humans traveling to multiple destinations without, at least in the near term, actually landing in these challenging gravity wells. Whereas NASA and other space agencies have pioneered the robotic exploration of most of the deep gravity wells in our solar system, mostly from near orbit, those explorations have been handicapped by latencies that largely prevent human explorers from being able to directly experience these venues, and to apply their cognition at those locations.

In this paper, we suggest that telerobotic control of surface assets on the Moon from a nearby venue in free space can be a powerful near-term strategy for bringing human cognition and decision making to lunar exploration, without actually putting humans on-site. This is a new paradigm for human-robot cooperation, wherein astronauts are used to operate robots that are not in their immediate vicinity though will experience, via telepresence, virtual exploration with them. As noted, putting an astronaut at a telerobotic control node within about $75,000 \mathrm{~km}$ of a lunar surface site allows this kind of intimate interaction. Such a node would require well-proven technologies for freespace habitation and life support, largely derived from developments on the ISS. Astronauts occupying an on-orbit 
habitat in free space would control surface robots with low latency, offering a telepresence-like experience without actually being very expensively on-site along with the robots.

It is helpful to evaluate siting options for such on-orbit lunar telerobotic control.

\section{A. Lunar Orbit}

Low lunar orbit (LLO) might be the obvious choice for such a control node, flying over the lunar surface at distances that would ensure extremely low latency - of order tens of milliseconds. But such a venue presents serious difficulties. Most low lunar orbits are dynamically unstable. Even for a highly stability-optimized $50 \mathrm{~km}$ near-polar orbit, LRO requires $150 \mathrm{~m} / \mathrm{s}$ of delta-V per year for orbit maintenance. Lack of stationkeeping propulsion would have led to impacting the surface after just a few months. Moreover, such an LLO orbit allows contact with individual surface assets only briefly, which would limit the operational efficiency of astronaut robotic teleoperators there. Such an orbit would not be sunsynchronous, with the habitat frequently shadowed, and power systems would rely heavily on batteries. A high lunar orbit presents different opportunities and problems. For example, a highly elliptical "frozen" orbit could be arranged to spend most of the apolune over the exploration site, offering a Molniya-type of communication opportunity with relatively little shadowing. A constellation of such orbits has been proposed to provide a long-lasting communication relay system for the entire lunar surface. ${ }^{19,20}$ This may, however, be disadvantageous for a range of exploration sites across the surface if just one telerobotic control node is in this orbit. The fact that the nodes of these orbits vary with time means that transfer to and from the Earth, and to and from any particular lunar surface site can change dramatically in propulsion needs as the orbit evolves.

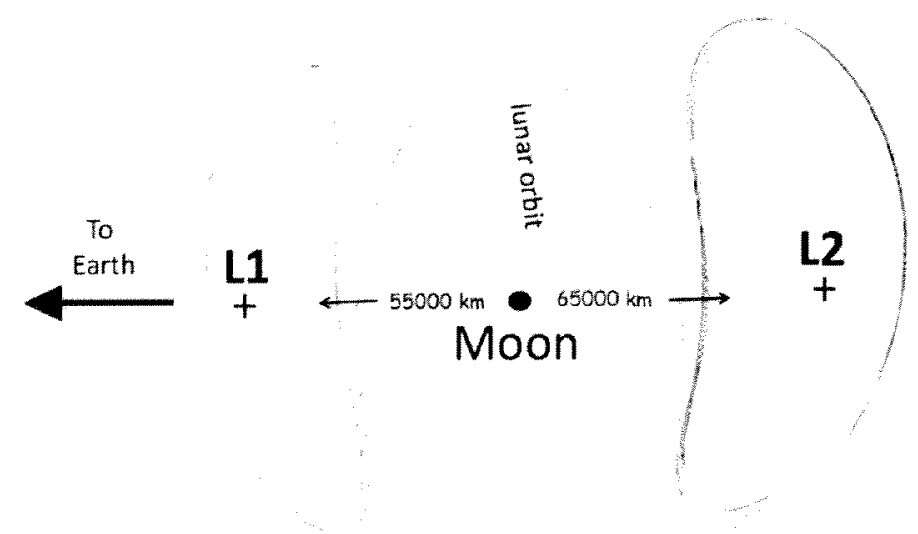

Figure 2. Orbits around EM L1 and L2. This figure shows a portion of the path of the NASA Artemis PI spacecraft as it orbited EM L1(projected onto the Moon's orbital plane -- XY), and was then transferred, by low-energy maneuver, to the complementary position around EM L2. This figure shows the spatial relationship of EM LI and L2 to the lunar surface. Such orbits, if used for a telerobotic control from a habitat, stay within the cognitive horizon of the near-or far-side of the lunar surface.

\section{B. Earth-Moon Lagrange Points}

Earth-Moon (EM) L1 and L2 orbits have, however, what we see to be strong potential for such control nodes, offering high performance. As shown in Figure 2, with the projection of the actual Pl orbit from the recent NASA Artemis mission in the lunar orbital plane, these orbits loop around the EM L1 and L2 points and, following the original concept for such a strategy by Farquhar $^{21,22}$ have been consistently considered as important venues for operations in cis-lunar space. These locations are attractive because of their proximity to the Moon, the nearly continuous illumination and line-of-sight to the Earth, the low cost of orbit stationkeeping, and the prospect of lowenergy transfers to other Lagrange points throughout the solar system. These qualities led the NASA Decadal Planning Team (DPT) ${ }^{23}$ and NASA Exploration Team (NExT) to identify them as sites for a "Gateway" habitation facility that would be a lynchpin in future exploration efforts throughout the solar system.

Direct insertion into these L1 and L2 orbits on a short duration trajectory (appropriate for human transport) requires about $3.6 \mathrm{~km} / \mathrm{s}$ delta-V from a $28^{\circ} \mathrm{LEO}$ orbit, but a longer-duration ballistic trajectory, which would be appropriate for cargo and for initially putting the habitat there, could be more economical. ${ }^{24}$ Such ballistic trajectories can cut the necessary trajectory correction maneuvers for orbit insertion by $700-800 \mathrm{~m} / \mathrm{s}$ after trans-lunar injection to about $50 \mathrm{~m} / \mathrm{s}$. Even the direct trajectory to $\mathrm{L} 1$ or $\mathrm{L} 2$ is several hundred $\mathrm{km} / \mathrm{s}$ lower than needed for LLO insertion and far lower, and hence less complex and propulsively expensive, than for actual landing on the lunar surface.

5

American Institute of Aeronautics and Astronautics 
As is evident from the scale in Figure 2, both $\mathrm{L} 1$ and $\mathrm{L} 2$ orbits are well within the cognitive horizon of the lunar surface and, in fact, having the control node a lot closer would offer few advantages. The orbital periods are roughly half a month. L1 offers continuous visibility to the lunar near side, while L2 offers continuous visibility to the lunar far side. As demonstrated by Artemis, transfer between L1 and L2 is possible with a modest investment in propulsion, on a time scale of a month or two. In this paper, we are neutral about the value of one Lagrange point over the other for a telerobotic control node, as the specific tasks will dictate optimal placement. The relative advantages and disadvantages of the two locations have been summarized in a recent essay. ${ }^{25}$ For teleoperation opportunities, the visibility of one entire hemisphere assures, in principle, that at least one sunlit site could always be accessible for telerobotic control.

Though not evident in Figure 2, the families of Lagrange point orbits have Z-components (out of the Moon's orbital plane). As such, a telerobotic node in these orbits would spend half the time above the orbital plane, and half the time below it. This leads to one of the few disadvantages of such orbits, which is that line-of-sight to the lunar poles, which may have high potential for in-situ resource utilization (ISRU) and long duration illumination is not continuous. Without a constellation of relay satellites (perhaps also in L1 or L2), which would increase latency somewhat, a given pole would be accessible to the $\mathrm{L} 1$ or $\mathrm{L} 2$ habitat only half the time. We note that communication with lunar polar outposts is considered problematical in general, as line-of-sight to the Earth is not continuous from those locations either.

In summary, EM L1 and L2 appear to be enabling sites for telepresence-level control of lunar surface robots. They offer communication latencies that are six times smaller than from the Earth, less than half a second. In that respect, they offer truly cognitive operations to the astronauts who are controlling those surface robots from the habitat there. Those locations offer continuous, uninterrupted access to either the lunar near- or far-side, with a roughly constant communication latency. The line-of-sight to the Earth is never blocked. Even at L2, with a halotype orbital topology, the Earth is always seen over the limb of the Moon. Solar power is largely uninterrupted, though solar eclipses lasting an hour or two can happen occasionally. Little orbit maintenance is required. Although these locations are not dynamically stable (which is actually an advantage in mitigation of debris) only several tens of $\mathrm{m} / \mathrm{s} / \mathrm{yr}$ of propulsion are needed to do the necessary stationkeeping. The orbits are fairly slow, so precision pointing of high bandwidth communication antennae at the habitat or at the client robot on the surface is not hard to manage. From these Lagrange point locations, in which one entire hemisphere of the lunar surface is visible, there are opportunities for simultaneous control of multiple sites on that hemisphere. This is of great value for lunar science and ISRU reconnaissance. These tasks are accomplished without putting humans into an expensive deep gravity well, at least in the near term.

\section{Habitats at Earth-Moon L1 and L2}

Habitats at EM L1 and L2 have been proposed as near-term destinations for human spaceflight beyond low Earth orbit, and offer a wealth of other services to space exploration. ${ }^{26}$ Those sites were evaluated as functional locales by the 2005 NASA Exploration Systems Architecture Study (ESAS), although were not incorporated in the eventual Constellation plan. On-orbit telerobotic control was not considered in that study. Near-term prospects for such habitation facilities will be reviewed in another paper by Thronson et al. at this meeting. The 2000 Decadal Planning Team recommended EM LI as a "Gateway" site, where large ships bound for Mars could be assembled and support for lunar surface operations could be provided. Such support could be in the form of depoting supplies for lunar surface installations, recognizing that, unlike from low lunar orbit, any site on the lunar surface is equally accessible from one of these Earth-Moon Lagrange points. By the same token, such a habitat could act as a propellant depot for cis-lunar vehicles, and perhaps as a refinery and storage facility for lunar ISRU products. The very small propulsion needs to move a payload back and forth from the highly favored operations sites for science spacecraft of Earth-Sun L1 and L2 to the far more astronaut-accessible Earth-Moon L1 or L2 sites (of order tens of meters per second), leads to the prospect of using such a habitat as a "job site" for servicing and construction of, for example, large space telescopes. ${ }^{27,28}$ In this respect, on-orbit control of lunar surface telerobots would be just one of many functions of such a habitat facility. Many of these habitat-local depoting and servicing functions would also be executed telerobotically from the habitat, in order to avoid EVA. The same telerobotic control station used for surface robot operations could be used for these local telerobotic operations.

\section{Extensibility of On-Orbit Telerobotic Control}

6

American Institute of Aeronautics and Astronautics 
One of the attractive aspects of on-orbit lunar surface telerobotics is that it develops a capability that can be extended to many other solar system sites. The prospect of doing on-orbit surface telerobotic control at Mars from Mars orbit (for example, from the roughly aerostationary Deimos) was originally proposed by Singer in 1977$1978^{29}$. This idea for Mars was developed further in a number of studies ${ }^{30}$ leading eventually to an intensive concept study on Human Exploration using Real-time Robotic Operations (HERRO) ${ }^{31,32}$, and also in independent concept studies with a similar strategy. ${ }^{3,34}$ The HERRO program was even considered as baselining an on-orbit strategy to do human telepresence on the surface of Venus. This idea is clearly extensible much farther, allowing low-latency human experience at sites that are overwhelmingly hostile to human visits. Consequently, we believe that on-orbit, low latency telerobotic control of surface assets is a novel and feasible approach to human exploration of the entire solar system. While the latency advantages of it for the Moon are only of order a few seconds compared to the Earth, proof of concept via lunar exploration would be instructive for sites at which the control latency would otherwise be far larger if carried out from the Earth.

\section{Candidate Lunar Tasks}

NASA's Constellation plan proposed to use telerobots locally outside of surface habitats to reduce the need for regular EVA. In this way, development plans for the Moon were seen to be largely telerobotic, though controlled

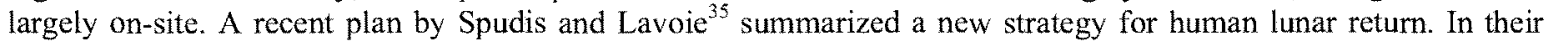
roadmap, site development and pre-emplacement would be based entirely on telerobotic activities commanded from the Earth, in preparation for eventual human visits to the lunar surface. This roadmap, as well as others, can be used to provide a set of notional tasks for which very low-latency telerobotic control could be useful. Some of these are

- Sampling, inspection, and rudimentary assaying of regolith and layering

- Manipulation and collection of samples for analysis or return

- Detailed site surveying and reconnaissance

- Groundbreaking for, assembly, servicing, and operation of ISRU test and mining facilities

- Scientific instrument emplacement, control, and maintenance

- Regolith grading, compacting, and "paving" for dust suppression

- Deployment of communication hubs and power stations

- Deployment of habitats

- Construction of regolith shield walls

For robotic recon in particular, a number of recent studies have recognized the power that such an effort would bring, for both science and technical return, before human visits. ${ }^{36,37,38}$ Such recon would essentially map out planned sorties prior to any EVA. This recon could be done on spatial scales that are impractically small for surveillance cameras in orbit. Whereas these studies have focused on recon work that could be done with telerobotic control from the Earth, probably for that reason little consideration was given for doing this work at lower latency. These studies emphasized visual inspection, with both high resolution and high dynamic range imagers, perhaps with microscopic capabilities. Even without any real dexterity, such mobile recon platforms could contribute strongly to later discovery. But adding low-latency dexterity, samples could be manipulated for higher-quality inspection, and rovers could be driven into more challenging locations.

It is clear that all of these tasks could, in principle, be fulfilled by telerobotic control from the Earth managed by a much larger number of teleoperators, each working slowly. Such operation would not be greatly benefitted from lessons of terrestrial applications of low latency telerobots. But the low latency that one would experience with control at a habitat at Earth-Moon L1 or L2 could offer greatly increased efficiency, and higher-quality work for those efforts specifically requiring a high level of dexterity and awareness. There may be tasks that a factor-of-six lower latency would actually enable. In particular, we note that lower latency teleoperation contributes substantially to risk mitigation, in that tasks that latency renders inherently dangerous, or just being at sites that might be considered dangerous, can be selected. Lunar sites that we have visited to date were selected specifically in order to be not dangerous. For example, it is understood that exploration of the South Pole Aiken Basin, which is a scientifically rich site, but one with very rough terrain, might benefit significantly from a low-latency telerobotic approach. In particular, for polar regions, terrain navigation will best follow routes that are illuminated, a task that will require real time decision making. 
With this in mind, it is important to closely coordinate the on-orbit, low-latency control one could achieve with an L1 or L2 habitat with control from the Earth, in order to effectively separate tasks that can benefit most from the

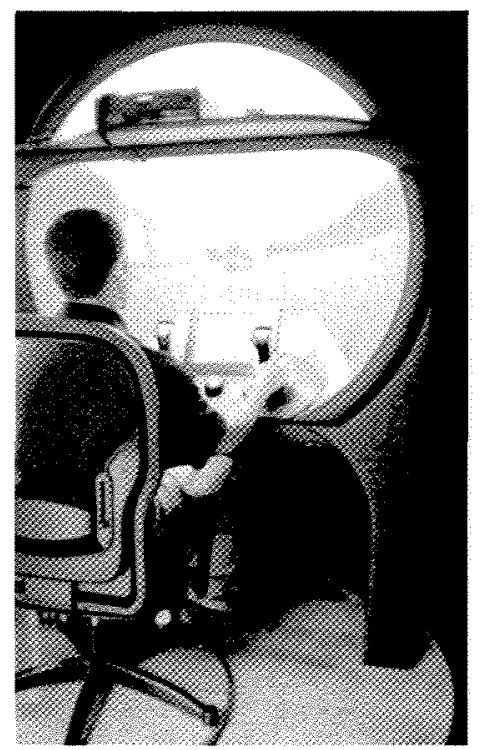

Figure 3. Representative telepresence station. This figure shows a proposed telepresence station for terrestrial telerobotic mining. Image from Penguin Automated Systems.

lower latency. It is worth considering that such an on-orbit control node would also be an excellent training ground for a future lunar surface astronaut who would eventually be in a position to control these same surface robot assets with essentially no latency from a habitat on the surface, and as a proving ground for those surface telerobotic control systems.

\section{On-Orbit Telerobotic Control Architecture}

\section{A. Architectural Concepts}

A great deal of effort has been expended on habitat architecture for deep space, and at the Earth-Moon Lagrange points in particular, but very little consideration has been given to the architectural requirements that telerobotic control would impose on such a habitat. We have experience controlling Dextre and Canadarm 2 from within the ISS, but it is likely that lunar operations will be more time consuming and intensive, such that more immersive architecture than a simple rack mounted control system would be required. For terrestrial telerobotic mining and drone piloting, operators are increasingly using multiple or large display screens, and ideas for scene-immersive wrap-around screens and stereo vision have been proposed. For driving lunar vehicles, perhaps with dexterous appendages attached, a "drivers seat" arrangement might be optimal, with both driving controls and manipulator controls in a convenient console (see Figure 3).

The way the architecture could best reflect and enable switching between multiple surface robots, perhaps at very different lunar surface sites, needs to be considered. With regard to the required habitat crew size, it would depend on the number of tasks to be controlled, and how those tasks would best be shared with controllers on Earth. It is likely that telerobotic operation from the habitat would take place on time scales of months, with the assumption that the skill of the operator in dealing with even the modest latency imposed would increase during this time. As such, the habitat would require requisite ECLSS, zero-g countermeasures, and radiation shielding. In other respects, the habitat can be very basic. This particular on-orbit telerobotic function does not require an airlock, or routine EVA capability. It would not demand multiple docking ports, a truss, or local manipulator tools for depot management. In this respect, on-orbit telerobotics can be one of the first functions of an evolving habitat.

The communication architecture is an important consideration. Such on-orbit control systems, offering high frame-rate video and perhaps even binocular video to the controller, will need of order $10 \mathrm{Mb} / \mathrm{s}$ uplink from the lunar surface, with control downlinks perhaps an order of magnitude smaller. As such, high frequencies are favored, and such high frequencies are not compromised by atmospheric conditions, as they might be on Earth or Mars. Sand Ka-band links should be considered, as might optical communication. As proposed for HERRO, a notional architecture would include a central communication station on the surface, ideally self-mobile, that can provide the main link to the on-orbit habitat, and act as a router for a local surface network composed of various pieces of robotic hardware in the immediate vicinity. These pieces would not need individual pointed antennae, but could work with far more manageable omnidirectional ones. The central communication station and the habitat itself will be linked to by directional antennae, with sizes of order $1 \mathrm{~m}$. This arrangement places relatively simply engineering constraints on the individual robotic units, especially with regard to communication systems. Such communication systems with the requisite bandwidth can be easily powered by modest solar panels. SCAWAG-consistent architectures relevant to EM LI/2 and the lunar surface, as well back to the Earth from the L1 and L2 Lagrange points have been reviewed in several places. ${ }^{39,40}$

Figure 4 shows a notional image of such a habitat (based largely on the Decadal Planning Team "Gateway" habitat concept). This habitat has a communication link to a central communication station that is surrounded by individual robotic units that are to be remotely controlled. Whereas this shows the habitat-to-surface communication link, it does not show the Earth-to-surface link (nor the Earth-to-habitat) link. These must be engineered not to 


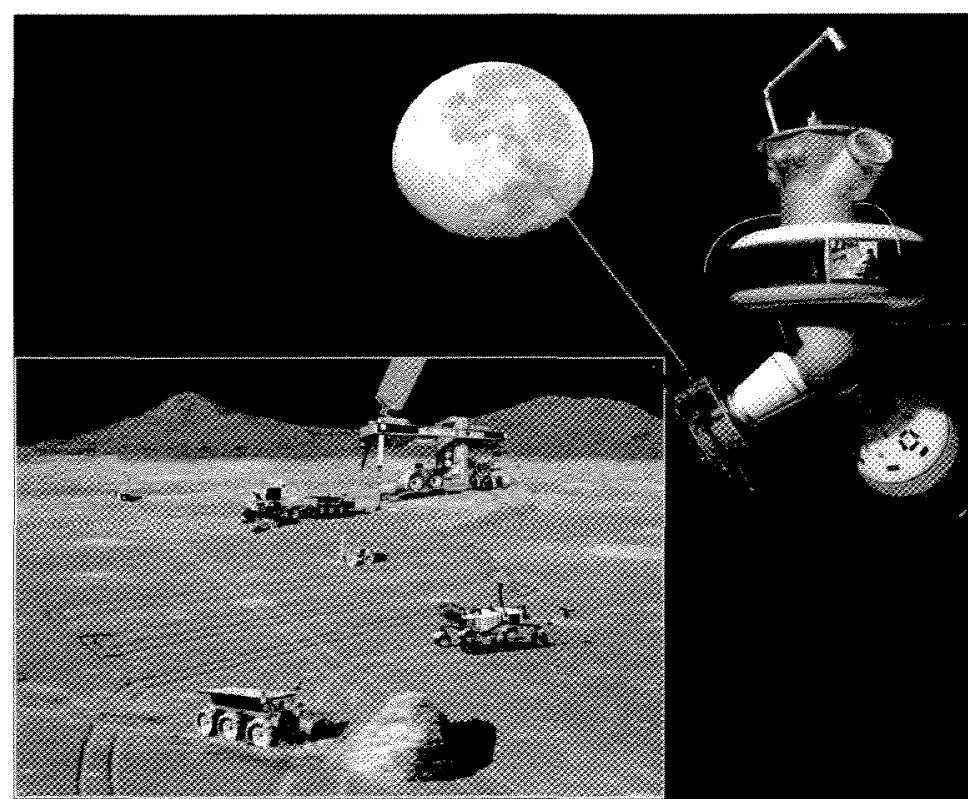

Figure 4. Notional on-orbit telerobotic control architecture. $A$ notional habitat at Earth-Moon L1 (or L2) is outfitted for telerobotic control at a lunar exploration site, about $400 \mathrm{~ms}$ of two-way latency away. The habitat is continuously illuminated, providing reliable power, and has full hemisphere visibility. Some portion of that hemisphere will always be sunlit, allowing robotic activity. While represented here schematically as an optical communication beam, $S$ or Ka-options will also meet bandwidth needs. Image adapted from NASA graphics. least large-scale regolith moving. Precision dexterous manipulators for doing lifting, aligning, and connecting are more complicated, but again are technologies that are driven strongly by terrestrial applications. Low latency telerobotic control of systems in LEO, and especially for servicing systems in GEO, will incentivize development of such space qualified units. The available robotic systems will dictate the tasks that would be undertaken. As to development of a $\mathrm{L} 1$ or $\mathrm{L} 2$ habitat, such a facility have strong design heritage from ISS, and will be a key to any work done in free-space beyond LEO. With regard to technology availability, the lessons from terrestrial, lowlatency telerobotic applications are an important strength. NASA doesn't need to wholly invent the technologies that are needed here.

\section{Conclusion}

On-orbit control of telerobots on the lunar surface can be an important strategy for near-term lunar exploration, allowing complex and detailed efforts on the lunar surface to be carried out with minimal latency and fully cognitive human involvement. It is an opportunity to achieve real telepresence for this work. Basically, latency mitigation is herein achieved by largely removing latency. The Earth-Moon L1 and L2 Lagrange points are high-quality venues from which to do this work, from habitats in orbits around those locations that are intended to provide value for many future exploration tasks. Development of such a habitat would exercise capabilities in long-duration human spaceflight beyond LEO and, beyond that, such telerobotic control of lunar surface agents could easily be one of the first tasks carried out at such a habitat. In this respect, such telerobotic control is highly consistent with a near-term space exploration strategy that does not require sending humans into expensive, deep gravity wells, while bringing human cognitive capabilities and to robotic agents on the lunar surface.

These Lagrange points offer continuous communication with one hemisphere of the lunar surface and, as such, provide an important "high camp" for control of telerobotic agents at multiple surface sites. At that Lagrange point location, the habitat is in almost continual sunlight, with unobscured communication lines of sight back to the Earth. 
Stationkeeping requirements are modest, and opportunities for Earth-return are uninterrupted, involving consistent propulsive requirements. The extensibility of such on-orbit telerobotic control is exciting, as such control could presage and support teleoperation from orbit on the surface of Mars, and support human telepresence at far more hostile sites. In that respect, on-orbit telerobotic control serves to greatly expand the possible destinations for human experience in the solar system. Development of a capability for lunar surface telerobotic control serves as a prototype for such future missions.

\section{Acknowledgments}

We appreciate discussions with members of the HERRO team at the NASA Glenn Research Center, George Schmidt and Geoff Landis in particular, as well as Gregg Podnar, Greg Baiden, Bill Clancey, Mike Raftery, Kip Hodges, and David Mindell.

\section{References}

${ }^{1}$ Adams, J.L. "An Investigation of the Effects of the Time Lag Due to Long Transmission Distance Upon Remote Control", NASA TN D-1351, 1962.

${ }^{2}$ Ferrell, W.R. "Remote Manipulation with Transmission Delay", IEEE Transactions in Human Factors in Electronics, Vol. 6, No. 1, pp. 24-32. 1965.

3 Sheridan, T., "Space Teleoperation Through Time Delay: Review and Prognosis", IEEE Transactions of Robotics and Automation, Vol. 9, No. 5, pp. 592-605, 1993

${ }^{4}$ Lane, J., Carignan, C. and Akin, D. "Time Delay and Communication Bandwidth Limitation on Telerobotic Control" SPIE Telemanipulator and Telepresence VII Conference, 2000

${ }^{5}$ Elfes, A., Dolan, J., Podnar,G., Mau, S., and Bergerman, M. Proceedings of the AAAI Spring Symposium, pp. 104-113, March 2006.

"F. Heger, F., Hiatt, L., Sellner, B., Simmons, R., and Singh, S. "Results in Sliding Autonomy for Multi-robot Spatial Assembly." 8th International Symposium on Artificial Intelligence, Robotics and Automation in Space Munich, Germany, September, 2005.

7 Vertisi, J. "Seeing Like a Rover: Embodied Experience on the Mars Exploration Rover Mission" 2008 Computer-Human Interaction (CHI) Conference, (DOI: 10.1145/1358628.1358709) [cited 1 August 2011]

"Clancy, W. "Becoming a Rover" 2009, http:/homepage.mac.com/WJClancey/\%7EWJClancey/MERScientistsIdentity.pdf. [cited 1 August 2011].

Anvari, M. et al. "The Impact of Latency on Surgical Precision and Task Completion During Robotic-Assisted Remote Telepresence Surgery" Computer Aided Surgery, Vol. 10, No. 2, pp. 93-99, March 2005.

${ }^{10} \mathrm{Kim}$, T., Zimmerman, P., Wade, M, and Weiss, C. "The Effect of Delayed Visual Feedback on Telerobotic Surgery" Surgical Endoscopy 19: pp. 683-686, 2005.

"Lum, M., et al. "TeleRobotic Fundamentals of Laparoscopic Surgery (FLS): Effects of Time Delay - Pilot Study" 30th Annual International IEEE EMBS Conference Vancouver, British Columbia, Canada, August 20-24, 2008.

${ }^{12}$ Charles R. Doarn, Mehran Anvari, Thomas Low and Timothy J. Broderick "Evaluation of Teleoperated Surgical Robots in an Enclosed Undersea Environment" Telemedicine and e-Health Vol 15, No. 4, pp. 325-335, May 2009.

${ }^{13}$ Baiden, G., "A Study of Underground Mine Automation", Ph.D. Thesis, Department of Mining and Metallurgical Engineering, McGill University, March 1993.

${ }^{14}$ Nourbakhsh, 1. et al. "Human-Robot Teaming for Search and Rescue." Pervasive Computing, Vol. 4, No. 1, pp. 72-78, Jan-Mar 2005.

is Hirzinger, G., Brunner, B., Dietrich, J., and Heindl, J. "ROTEX - The First Remotely Controlled Robot in Space", 1994 IEEE International Conference on Robotics and Automation.

${ }^{16}$ Pressman, A., Welty, L., Karniel, A., and Mussa-Ivaldi, F. "Perception of Delayed Stiffness" The International Journal of Robotics Research Vol. 26, pp. 1191-1203, 2007.

${ }^{17}$ Hu, J., Thompson, J., Ren, J, and Sheridan, T. "Investigatons into Performance of Minimally Invasive Telesurgery with Feedback Time Delays" Presence: Teleoperators and Virtual Environments, Vol. 9, No. 4, pp. 369-382, 2000.

18 Augustine, N. et al. "Seeking a Human Spaceflight Program Worthy of a Great Nation: Review of Human Spaceflight Plans Committee", Office of Science \& Technology Policy, 2009.

${ }^{19}$ Coffey, S., Deprit, A., and Deprit, E., "Frozen Orbits for Satellites Close to an Earth-Like Planet," Celestial Mechanics and Dynamical Astronomy, Vol. 59, No. 1, pp. 37 72, 1994.

${ }^{20}$ Ely and Lieb "Constellation of Elliptical Inclined Lunar Orbits Providing Polar and Global Coverage." AAS/AlAA Astrodynamicy Specialist Conferenee, Lake Tahoe, CA. August 7-11,2005.

21 Farquhar, R. W., "The Control and Use of Libration-Point Satellites", NASA Technical Report R-346, Washington, D. C., Sept. 1970 
${ }^{22}$ Farquhar R W. "A Halo-Orbit Lunar Station" Astronaut. Aeronaut. J, Vol. 10, pp. 59-63, 1972.

${ }^{23}$ Garber, S., and Asner, G. "NASA's Decadal Planning Team and the Policy Formulation of the Vision for Space Exploration" NASA History Office http//history.nasa.gov/DPT/DPT.htm [cited 1 August 2011]

${ }^{24}$ Parker, J. "Targeting Low-Energy Ballistic Lunar Transfers" American Astronautical Society (AAS) George H. Born Symposium, Boulder, Colorado. May 13, 2010.

${ }^{25}$ Lester, D. "Picking Sides in Cislunar Space" The Space Review http://www thespacereview.com/article/1808/1 March 28, 2011 [cited 1 August 2011].

${ }^{26}$ Thronson, H., Lester, D., Moe, R., and Sullivan, G. "Review of US Concepts for Post-ISS Space Habitation Facilities and Future Opportunities" AlAA Space 2010

${ }^{27}$ Lester, D. "Servicing and Lagrange Point Operations for Astronomy" International Workshop on On-Orbit Satellite Servicing NASA Goddard Space Flight Center, March 2010.

${ }^{28}$ Lester, D., Budinoff, J., and Lillie, C. "Large Infrared Telescopes in the Exploration Era-SAFIR" SPIE Proceedings 6687-18, UV/Optical/IR Space Telescopes: Innovative Technologies and Concepts III, 2007.

${ }^{29}$ Singer, S. "The PH-D Proposal: A Manned Mission to Phobos and Deimos," AAS 81-231 The Case for Mars, Penelope Boston, editor, 1984, pp. 39-65; paper presented at the Case For Mars conference, Boulder, Colorado, April 29-May 2, 1981.

${ }^{30}$ Landis, G. "Robots and Humans: Synergy in Planetary Exploration" Acta Astronautica Vol. 55, pp. 985-990, 2004.

${ }^{31}$ Schmidt, G., Landis, G., and Oleson, S. "Science Rationale for Flexible Path: A Robotically Intensive Crew-Based Exploration Strategy for the $21^{\text {st }}$ Century" International Astronomical Congress 1AC-10A5.2.5, 2010.

${ }^{32}$ Schmidt, G., Landis, G., Oleson, S., Borowski, S., and Krasowski, M. "HERRO: A Science-Oriented Strategy for Crewed Missions Beyond LEO" 48th ALAA Aerospace Sciences Meeting Including the New Horizons Fontm and Aerospace Exposition, AIAA 2010-629, 4 - 7 January 2010, Orlando, Florida.

33 Podnar, G., Dolan, J., and Elfes, A. "Telesupervised Robotic Systems and the Human Exploration of Mars" Journal of Cosmology, Vol. 12, pp. 4058 4067, 2010.

${ }^{34}$ Bosquillon de Frescheville, F., Martin, S., Policella, N., Patterson, M., Aiple, M., Steele, P. "Set-Up and Validation of METERON End-End Network for Robotic Experiments" ESA/ESTEC ASTRA 2011 Conference, 12-14 April 2011.

${ }^{35}$ Spudis, P., and Lavoie, T. "Mission and Implementation of an Affordable Lunar Retum," December 19, 2010 http://www.spudislunarresources.com/Papers/Affordable Lunar Base.pdf. [Cited 1 August 2011)

${ }^{36}$ Fong, T. et al. "Assessment of Robotic Recon for Human Exploration of the Moon" Acta Astronautica, Vol. 67, pp. 1176$1188,2010$.

${ }^{37}$ Deans, A., et al. "Robotic Scouting for Human Exploration" AIAA Space 2009 Conference \& Exposition, 2009-6781.

${ }^{38}$ Schreckenghost, D, Fong, T., Milam, T., and Utz, H. 2009 Performance Metrics for Intelligent Systems Workshop (PERMIS 09) Gaithersburg, MD.

39 "NASA Space Communication and Navigation Architecture Recommendations for 2005-2030" NASA Space Communication Architecture Working Group (SCAWG), 15 May 2006

${ }^{40}$ Schier, J., Rush, J., Williams, W., and Vrotsos, P. "Space Communication Architecture Supporting Exploration and Science: Plans and Studies for 2010-2030" ALAA 1st Space Exploration Conference, 2005. 